\title{
Inside the 2016 American Society of Clinical Oncology Genitourinary Cancers Symposium: part 1 - kidney cancer
}

\author{
Sebastiano Buti ${ }^{1}$, Chiara Ciccarese ${ }^{2}$, Roberto lacovelli², Melissa Bersanelli', \\ Marina Scarpelli ${ }^{3}$, Antonio Lopez-Beltran ${ }^{4}$, Liang Cheng ${ }^{5}$, Rodolfo Montironi ${ }^{3}$, \\ Giampaolo Tortora² \& Francesco Massari*,6
}

\section{The American Society of Clinical Oncology Genitourinary Cancers Symposium, Moscone West Building, San Francisco, CA, USA, 7-9 January 2016}

The American Society of Clinical Oncology (ASCO) Genitourinary Cancers Symposium, held in San Francisco (CA, USA), from 7 to 9 January 2016, focused on 'patient-centric care: translating research to results'. Every year, this meeting is a must for anyone studying genitourinary tumors to keep abreast of the most recent innovations in this field, exchange views on behaviors customarily adopted in daily clinical practice, and discuss future topics of scientific research. This two-part report highlights the key themes presented at the 2016 ASCO Genitourinary Cancers Symposium, with part 1 reporting the main novelties of kidney cancer and part 2 discussing the most relevant issues which have emerged for bladder and prostate tumors.

First draft submitted: 27 February 2016; Accepted for publication: 1 June 2016; Published online: 17 June 2016

\section{Renal cell carcinoma: the second-line revolution}

In 2015, two new treatment options, nivolumab and cabozantinib, entered the therapeutic armamentarium of pretreated metastatic renal cell carcinoma (mRCC) patients after the demonstration of improved outcomes over the standard second-line everolimus in randomized Phase III studies (CheckMate 025 and METEOR trials, respectively). The PD-1 checkpoint inhibitor, nivolumab, significantly improved overall survival (OS: 25.0 vs 19.6 months; hazard ratio [HR]: 0.73) regardless of PD-L1 expression, objective response rate (ORR: 25 vs 5\%) with a peculiar durability of responses and quality of life with an excellent safety profile [1]. Likewise, cabozantinib, an oral multi-targeted tyrosine kinase inhibitor (TKI) of VEGFR, AXL and MET, led to a significant prolongation of progression-free survival (PFS: 7.4 vs 3.8 months; HR: 0.58 ) and ORR (21 vs 5\%) with an acceptable safety profile among mRCC patients progressed after VEGFR-targeted therapy [2]. At the present meeting, subgroup analyses of both these trials were presented.

'Medical Oncology Unit, University Hospital of Parma, Italy

${ }^{2}$ Medical Oncology, Azienda Ospedaliera Universitaria Integrata, University of Verona, Verona, Italy

${ }^{3}$ Section of Pathological Anatomy, Polytechnic University of the Marche Region, School of Medicine, AOU Ospedali Riuniti, Ancona,

Italy

${ }^{4}$ Department of Surgery, Cordoba University Medical School, Cordoba, Spain

${ }^{5}$ Department of Pathology \& Laboratory Medicine, Indiana University School of Medicine, Indianapolis, IN, USA

${ }^{6}$ Division of Oncology, S.Orsola-Malpighi Hospital, Bologna, Italy

*Author for correspondence: fmassari79@gmail.com

\section{KEYWORDS}

- biomarkers • genitourinary cancer $\bullet$ targeted therapy 
Dr Motzer (Memorial Sloan-Kettering Cancer Center [MSKCC], NY, USA) confirmed the consistent OS and ORR benefit of nivolumab across key subgroups: prognostic risk groups, number and sites of metastases and prior therapies [3]. In particular, nivolumab retained the OS improvement in each different risk category based on MSKCC and Heng criteria, with an impressive separation of the OS curves between nivolumab and everolimus in the intermediate- and poor-risk groups and a noteworthy high benefit for nivolumab in the subgroup of MSKCC poor-risk patients (median OS [mOS]: 15.3 vs 7.9 months; HR: 0.48; 95\% CI: 0.32-0.70). Despite the MSKCC risk assessment model was defined for mRCC in the era of immunotherapy (IL-2 and IFN- $\alpha$ ) while the Heng criteria were validated in the context of anti-VEGFR-targeted therapies, the latter prognostic score system includes factors more closely related to inflammation (i.e., neutrophilia, thrombocytosis). Thus, the need to establish and validate prognostic criteria tailored for the novel immune-modulatory agents is emerging. Moreover, nivolumab maintained its OS benefit over everolimus regardless of the number of metastases (considering patients with one vs two or more metastatic sites) and the presence of bone or liver metastases (mOS: 18.5 months with nivolumab vs 13.8 months with everolimus [HR: 0.72; 95\% CI: 0.47-1.09] in bone metastatic patients; 18.3 vs 16.0 months [HR: 0.81 ; 95\% CI: 0.55-1.18] in case of liver metastases), reiterating a significant activity of nivolumab also in case of clinical features commonly associated to a worse prognosis (multiple metastases, especially in bone and liver).

Regarding the impact of a previous therapy on the efficacy of the treatment, nivolumab was superior to everolimus in both subgroups of patients previously treated with sunitinib (slightly more than $60 \%$ of study population mOS: 23.6 vs 19.8 months; HR: 0.81; with 61\% of patients alive at 18 months with nivolumab compared with 54\% with everolimus) and pazopanib (mOS: not reached with nivolumab vs 17.6 months with everolimus; HR: 0.60; and proportions of survivors at 18 months similar to those previously reported - 63 vs $49 \%$ ). Finally, a survival advantage for nivolumab over everolimus was also demonstrated irrespective of the duration of first-line therapy (less vs 6 or more months) and of prior anti-angiogenic therapies (one vs two). In particular, nivolumab showed a clear benefit in the subgroup of patients (nearly $70 \%)$ treated with only one prior VEGFRTKI therapy before enrollment (mOS: 23.6 vs 19.9 months; HR: 0.79; 95\% CI: 0.63-0.99), emphasizing the potential role of nivolumab as second-line therapy.

Similar results favoring nivolumab were reported across the same key subgroups with respect to the ORR. The responses, higher for nivolumab compared with everolimus in all subgroups, were very similar across the different categories examined.

On the other hand, Dr Escudier (Institut Gustave Roussy, Villejuif, France) established the persistent benefit of cabozantinib over everolimus in term of PFS across key subgroups defined by baseline features, including MSKCC risk groups and patients Eastern Cooperative Oncology Group performance status, metastatic organ involvement and tumor burden and prior therapies (type and duration of prior VEGFRTKIs and prior anti-PD-1 therapy) [4]. More in details, as concern the prognostic groups, the superiority of cabozantinib was particularly evident in patients with good performance status (Eastern Cooperative Oncology Group performance status 0 vs 1 ) and fewer MSKCC risk factors (HR: 0.51, 0.47 and 0.70 in good, intermediate and poor MSKCC risk groups, respectively). However, slightly in contrast with these results, cabozantinib showed higher activity over everolimus in high tumor burden disease (sum of target lesion diameters $>65 \mathrm{~mm}$, two or more involved organs) compared with low disease burden patients.

Interestingly, the peculiar tropism of cabozantinib for bones was indirectly confirmed by the evidence of a greater benefit with this drug in patients with both visceral and bone metastases (median PFS [mPFS]: 5.6 vs 1.9 months; HR: 0.26). To further support this hypothesis, the subgroup analysis selected by sites of tumor metastases strongly favored cabozantinib over everolimus in patients with bone metastases (mPFS: 7.4 vs 2.7 months; HR: 0.33).

Regarding the impact of prior systemic therapy in affecting treatment efficacy, cabozantinib remained active even after the second line of treatment (the PFS of patients treated with one vs two prior VEGFR-TKIs was absolutely similar, 7.4 months in both subgroups). Moreover, the longer was period of first VEGFR-TKIs (< vs $\geq 6$ months), the longer was PFS for cabozantinib (5.6 vs 9.0 months), in contrast to what observed 
with everolimus, suggesting that the initial response to TKIs could be predictive of cabozantinib activity. In particular, in case of disease refractory to VEGFR-TKIs (time to progression on last prior VEGFR-TKI less than 3 months) there was a trend in favor of cabozantinib (mPFS: 5.5 vs 3.7 months; HR: 0.67).

A remarkably long PFS was noted in patients receiving cabozantinb after failure of first-line sunitinib therapy (mPFS: 9.1 vs 3.7 months with cabozantinib vs everolimus; HR: 0.43 ), while the PFS advantage with cabozantinib over everolimus was minor after treatment with pazopanib (mPFS: $7.4 \mathrm{~s}$ vs 5.1 months; HR: 0.67). In the small subgroup of patients $(\mathrm{n}=32)$ treated with prior PD-1/PD-L1 immunotherapy, a strong PFS benefit in favor of cabozantinib (HR: 0.22) supports further investigations in this setting.

As expected, the results of these two studies have further enlivened the debate on the choice of the appropriate second-line therapy for kidney cancer: immunotherapy invaded the historical struggle between VEGFR-TKIs and mTOR inhibitors, further increasing the 'embarrassment of the rich'. Definitely, the goal for the future is represented by the identification of response predictors to each specific treatment.

A potential clinical factor nullifying for second-line treatment choice could be the reason for discontinuation (progression vs toxicity) of first-line VEGFR-targeted therapy. Dr De Velasco (Dana-Farber Cancer Institute, MA, USA) reported the results of a multicentric retrospective study, evaluating whether clinical outcomes differ in mRCC patients receiving second-line targeted therapy depending on the reason for first-line treatment discontinuation [5] . Compared with discontinuation of first-line VEGFR-TKI due to disease progression (77\%; $\mathrm{n}=866$ ), patients who stopped treatment of toxicity (about $20 \%$ of cases $-\mathrm{n}=208$ ) had longer drug-free interval between first- and second-line (1.4 vs 0.7 months; $\mathrm{p}<0.001$ ), greater clinical benefit (complete or partial response and stable disease) in second line (68.5 vs $56.5 \%$; OR: 1.58 [95\% CI: $1.07-2.35] ; \mathrm{p}=0.023)$, greater probability to stop second-line treatment because of toxicity ( 14.6 vs $29.3 \%$; p < 0.0001), and longer OS from the start of second-line (17.4 vs 11.2 months; HR: 0.69 [95\% CI: 0.56-0.84]; $\mathrm{p}=0.0002-$ adjusted for type of therapy, time to initiation of second-line, Heng risk group and number of metastases at second-line therapy). Those patients who started a second line due to toxicity lived longer regardless of the king of second-line treatment type (VEGFR-TKIs or mTOR inhibitors) compared with patients who interrupted treatment for disease progression.

Certainly, besides clinical factors (features related to the patient, the tumor, prior therapies), efforts are required to identify specific biomarkers with prognostic and/or predictive impact. Attention is now focused on the role of the hosts' immune system in promoting/hindering RCC development and progression.

An interesting analysis presented by Ghatalia (Fox Chase Cancer Center, PA, USA) remarked the potential prognostic role of intratumoral immune cell infiltrates, identifying at multivariate analysis an independent association between high tumor infiltrating lymphocyte/plasma cell and macrophage (morphologically identified) with higher risk of recurrence $(p=0.0347)$ of localized ccRCC patients undergoing surgery [6]. Similarly, Dr Sekar (Emory University School of Medicine, GA, USA) developed a preoperative inflammatory marker prognostic score (RISC) for localized RCC patients undergoing nephrectomy, which considered preoperative C-reactive protein, albumin, erythrocyte sedimentation rate, corrected calcium and AST/ALT ratio) and seemed to act as a poor prognostic indicator of OS (increase in RISC was associated with a $32 \%$ increase in mortality (HR: 1.32; 95\% CI: 1.17-1.49; p < 0.001]) [7]. Moreover, the T-cell receptor (TCR) repertoire seemed to influence responses to VEGFR blockade in mRCC. Dr Ho (Mayo Clinic, AZ, USA) studied the TCR repertoire (TCR- $\gamma$ and TCR- $\beta$ CDR3 regions) evaluating TCR clonality and entropy (two measures of T-cell diversity) from pretreatment tumors of mRCC patients receiving pazopanib or placebo as first-line therapy [8]. Increased TCR entropy (leading to a broader T-cell population able of recognizing multiple different tumor antigens, with a low probability of tumor escape) was associated with a favorable prognosis in the placebo group compared with low entropy group (mPFS: 12.78 vs 4.40 months; $p=0.023$ ), whereas pazobanib attenuated this difference (suggesting that VEGRF-inhibition efficacy was independent of TCR entropy). Finally, Dr Voss (MSKCC) presented a promising genomic study of whole exome and transcriptome (RNAseq) sequencing of RCC samples from patients treated with nivolumab; it revealed that, albeit in a small cohort (seven mRCC patients), the expression 
of tumor neoantigens resulted from nonsynonymous somatic mutations correlated with objective response, assuming a predictive role of treatment benefit from nivolumab [9].

\section{Conclusion}

Although no studies were presented that can change clinical practice in kidney cancer, some topics may be considered of interest for future strategies. Subgroup analyses did not show a better outcome for nivolumab or cabozantinib but they merely confirmed the prognostic role of well-known factors largely used in previous studies, such as the duration of first-line therapy and the MSKCC or the International Metastatic Renal Cell Carcinoma Database Consortium classifications. Considering the recent announcement that cabozantinib also increases the OS in second-line, a large use of this drug could be hypothesized in the future, despite its worse safety profile compared than nivolumab, an important limit in real-world clinical practice.

Financial \& competing interests disclosure

The authors have no relevant affiliations or financial involvement with any organization or entity with a financial interest in or financial conflict with the subject matter or materials discussed in the manuscript. This includes employment, consultancies, honoraria, stock ownership or options, expert testimony, grants or patents received or pending, or royalties.

No writing assistance was utilized in the production of this manuscript.

\section{References}

1 Motzer RJ, Escudier B, McDermott DF et al. Nivolumab versus everolimus in advanced renal-cell carcinoma. N. Engl. J. Med. 373(19), 1803-1813 (2015).

2 Choueiri TK, Escudier B, Powles T et al. Cabozantinib versus everolimus in advanced renal-cell carcinoma. N. Engl. J. Med. 373(19), 1814-1823 (2015).

3 Motzer RJ, Sharma P, McDermott DF et al. CheckMate 025 Phase III trial: outcomes by key baseline factors and prior therapy for nivolumab (NIVO) versus everolimus (EVE) in advanced renal cell carcinoma (RCC). J. Clin. Oncol. 34(Suppl. 2S), Abstract 498 (2016).

4 Escudier BJ, Motzer RJ, Powles T et al. Subgroup analyses of METEOR, a randomized Phase 3 trial of cabozantinib versus everolimus in patients (pts) with advanced renal cell carcinoma (RCC). J. Clin. Oncol. 34(Suppl. 2S), Abstract 499 (2016).

5 De Velasco G, Xie W, Donskov F et al. Discontinuing VEGF-targeted therapy (VEGF-TT) for progression versus toxicity impacts outcomes of second-line therapies in metastatic renal cell carcinoma (mRCC). J. Clin. Oncol. 34(Suppl. 2S), Abstract 503 (2016).

6 Ghatalia P, Gordetsky J, Bae S et al. Association of a combined panel of tumor infiltrating lymphocytes, plasma cells, and macrophages with recurrence of localized clear cell (cc) renal cell carcinoma (RCC) undergoing surgery. J. Clin. Oncol. 34(Suppl. 2S), Abstract 502 (2016).
7 Sekar RR, Patil D, Pearl J et al. A novel preoperative inflammatory marker prognostic score in patients with clear cell and non-clear cell renal cell carcinoma. J. Clin. Oncol. 34(Suppl 2S), Abstract 530 (2016).

8 Ho TH, Gagnon RC, Liu Y et al. T-cell receptor (TCR) repertoire in metastatic renal cell carcinoma (RCC) patients treated with first-line vascular endothelial growth factor receptor blockade. J. Clin. Oncol. 34(Suppl. 2S), Abstract 501 (2016).

9 Voss MH, Hellmann MD, Chen Y et al. Mutation burden and tumor neoantigens in RCC patients (pts) treated with nivolumab. J. Clin. Oncol. 34(Suppl. 2S), Abstract 514 (2016). 